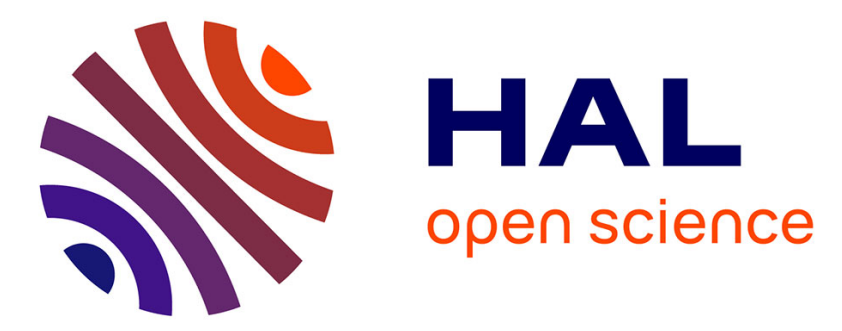

\title{
Nanopsychiatry-the potential role of nanotechnologies in the future of psychiatry: a systematic review.
}

\author{
Guillaume Fond, Alexandra Macgregor, Stéphanie Miot
}

\section{To cite this version:}

Guillaume Fond, Alexandra Macgregor, Stéphanie Miot. Nanopsychiatry-the potential role of nanotechnologies in the future of psychiatry: a systematic review.. European Neuropsychopharmacology, 2013, 23 (9), pp.1067-71. 10.1016/j.euroneuro.2012.10.016 . inserm-00759948

\section{HAL Id: inserm-00759948 https://www.hal.inserm.fr/inserm-00759948}

Submitted on 3 Dec 2012

HAL is a multi-disciplinary open access archive for the deposit and dissemination of scientific research documents, whether they are published or not. The documents may come from teaching and research institutions in France or abroad, or from public or private research centers.
L'archive ouverte pluridisciplinaire HAL, est destinée au dépôt et à la diffusion de documents scientifiques de niveau recherche, publiés ou non, émanant des établissements d'enseignement et de recherche français ou étrangers, des laboratoires publics ou privés. 
Nanopsychiatry.

The potential role of nanotechnologies in the future of psychiatry. A systematic review.

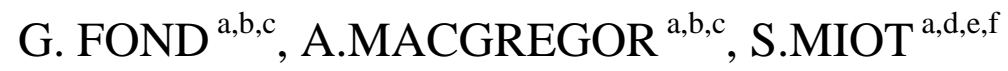

${ }^{\mathrm{a}}$ Université Montpellier 1, Montpellier F-34000, France

b Institut National de Santé et de Recherche Médicale INSERM, U1061, Montpellier F-34093, France

c Service universitaire de psychiatrie adulte, Hôpital La Colombière/CHRU de Montpellier, F-34000, France

d Institut National de Santé et de Recherche Médicale INSERM, U952, Physiopathologie des Maladies du Système nerveux Central, 9 Quai St Bernard, 75005 Paris, France

${ }^{\mathrm{e}}$ CNRS UMR 7224, 9 Quai St Bernard, 75005 Paris, France

${ }^{\mathrm{f}}$ UPMC, Univ Paris 06, F-75005 Paris, France

corresponding author

Dr Guillaume Fond

Chef de Clinique - Assistant des Hôpitaux

Université Montpellier 1 et Inserm U1061

Service Universitaire de Psychiatrie Adulte (Pr. JP. Boulenger),

Hôpital La Colombiere CHU Montpellier

39, avenue Charles Flahault 34295 Montpellier cedex 05

tel: + 33467339702 fax: + 33467338995

guillaume.fond@gmail.com

word count 2742 
Abstract.

Nanomedicine is defined as the area using nanotechnology's concepts for the benefit of human beings' health and well being.

In this article, we aimed to provide an overview of areas where nanotechnology is applied and how they could be extended to care for psychiatric illnesses.

The main applications of nanotechnology in psychiatry are (i) pharmacology. There are two main difficulties in neuropharmacology: drugs have to pass the blood brain barrier and then to be internalized by targeted cells. Nanoparticles could increase drugs bioavailability and pharmacokinetics, especially improving safety and efficacy of psychotropic drugs. Liposomes, nanosomes, nanoparticle polymers, nanobubbles are some examples of this targeted drug delivery. Nanotechnologies could also add new pharmacological properties, like nanohells and dendrimers (ii) living analysis. Nanotechnology provides technical assistance to in vivo imaging or metabolome analysis (iii) central nervous system modeling. Research teams have succeded to modelize inorganic synapses and mimick synaptic behavior, a step essential for further creation of artificial neural systems. Some nanoparticle assemblies present the same small worlds and free-scale networks architecture as cortical neural networks. Nanotechnologies and quantum physics could be used to create models of artificial intelligence and mental illnesses.

We are not about to see a concrete application of nanomedicine in daily psychiatric practice. Even if nanotechnologies are promising, their safety is still inconsistent and this must be kept in mind. However, it seems essential that psychiatrists do not forsake this area of research the perspectives of which could be decisive in the field of mental illness.

Key words : nanomedicine, nanocarriers, imaging, metabolome, intelligence. 


\section{Introduction}

Nanomedicine is defined as the area using the concepts of nanotechnology for the benefit of humans' health and well being (Surendiran et al., 2009; Wang et al., 2011). Nanoparticles, from 1 to 100 nanometers, are here designated and used as diagnostic and therapeutic tools, or for biomedical research (Roney et al., 2005). The field of nanotechnology opens new avenues unsuspected a few years ago, both in the field of oncology, pharmacology, tissue regeneration, biosensors ... but also the modeling of complex systems such as the central nervous system. The advantages of nanoparticles seem numerous, as crossing biological barriers and increasing the specificity of drug treatments by accumulating them in a specific site of the body, and reducing side effects (Wang et al., 2011).

Nanomedicine research has expanded greatly in the last decade, with only a few dozen publications in the 1980's to more than 32,700 articles referenced in Medline to this day(last search July 20, 2012). However, very little data concerning research and development in psychiatry or mental health has yet been published.

The aim of our study is therefore to provide an overview of the nanotechnology application areas that could concern treatment of psychiatric illnesses.

We conducted a systematic review using the PRISMA criteria (preferred reporting items for systematic reviews and meta-analysis). Inclusion criteria. Articles were specified in advance, they concerned all studies describing the development of nanotechnology in psychiatry, mental health or neurosciences. No limits on language, publication date or publication status were imposed. Articles were identified in three research bases, Medline (1966-present), Web of Science (1975-present) and Cochrane. The last search was launched July 20, 2012. The research paradigm was: "(nanoparticles OR nanotechnology OR nanomedicine) AND (Central Nervous System)". The reference list of each article was reviewed to add items of potential interest. The research was done by two independent authors (G.F and S.M). 1135 items were found. 15 were selected after reading the abstracts. Disagreements over the selection of articles were resolved by consensus between the authors. 61 items were added after reviewing the references of these articles.

(i) pharmacology. Nanoparticles could increase the bioavailability and pharmacokinetics of drugs. Liposomes, virosomes, polymeric nanoparticles and nanosuspensions are some examples of this targeted drug delivery. New therapeutic approaches may be explored: 
nanoparticles are different from traditional small molecules in structure and may possess innovative therapeutic properties.

(ii) the analysis of living organisms. Nanotechnology provides technical assistance to in vivo imaging or metabolome analysis.

(iii) modeling the central nervous system (CNS). Assemblies of nanoparticles could be used to model artificial intelligence and potentially, mental illness.

Results

Pharmacology

One of the first issues of nanotechnology is to improve the safety and efficacy of psychotropic treatments.

Targeted delivery of the drug:

The entry of the drug into target cells remains a major challenge in pharmacology (Wang et al., 2011). Phagocytosis, the various types of endocytosis (clathrin-mediated, caveolaemediated or both), and macropinocytosis are now well known processes. Studies of these cellular internalization mechanisms have identified several factors responsible for their success. The particle size should preferably be between 10 and 100 nanometers. The particle shape is also important, the contact angle between the cell membrane and the particle has to be preferably less than 45 degrees. The rigidity of the particles is also a limiting factor. The surface charge will favor a certain type of endocytosis (Chertok et al., 2010). Finally internalization will also depend mainly on the type of chemical surface and the expression of receptors or specific ligands (Wang et al., 2011). These elements have an impact on the biodistribution and pharmacokinetics of drugs. In terms of psychotropic treatments, one limiting factor is superimposed: the passage of the blood-brain barrier (BBB) (Szebeni, 2011). Indeed, over $98 \%$ of low molecular weight molecules can not cross the BBB (Pardridge, 2006). Consequently, many neurological and psychiatric diseases remain inadequately treated. The ideal molecule to cross the BBB is a small, compact and lipophilic molecule (this parameter is measured by the charge distribution on the surface of the molecule) (Roney et al., 2005). The passage of the molecule into the peripheral circulation exposes it to enzymatic degradation by opsonization that can lead to its elimination before it has time to reach the brain, unless the molecule is considered as harmless by the recipient organism. Two basic paradigms have been proposed to allow the drug to reach it's target in the brain (Roney et al., 2005). The first proposes to develop molecules that can cross the BBB, which would then be 
"activated" by enzymes expressed in target cells of the brain. The major limitation of this paradigm is the low number of drug candidates and exploitable metabolic cascades. The molecules could be addressed via receptor endocytosis, but many receptors are not specific to a particular cell type. The drug would not be released specifically in target tissues, thus being responsible for side effects. The second paradigm seems to work around this problem, involving carriers, which can cross biological barriers, target a given cell type via a ligand expressed on their surface, and thus deliver the drug inside them. Nanotechnology offers the possibility to produce such carriers: nanocarriers, particles of small sizes that can be internalized by the cells.

Amongst these nanocarriers are liposomes, modeled in the 60s. These spherical nanoparticles composed of a lipid bilayer membrane are able of delivering drugs by targeting a specific cell, through their coupling to specific ligands (Surendiran et al., 2009). Similarly, the nanosomes are able to enter cells by endocytosis but without requiring specific ligand endocytosis (Surendiran et al., 2009). Nanotubes are also good candidates. Tubular structures made of graphite, they are relatively soluble and useful in the transport of certain drugs such as antibiotics or certain vaccines (Surendiran et al., 2009).

The polymeric nanoparticles also have many advantages: they have a strong capacity to transport molecules, thereby increasing the intracellular delivery. Their solid matrix protects the drug from the degradation device, mainly by allowing the molecule to escape recognition by the reticular endothelial system of macrophages, increasing the chances of the drug of reaching the brain (Szebeni, 2011; Wang et al., 2010). Finally polymeric particles are able to open the tight-junctions of the BBB, thus prolonging drug release (Roney et al., 2005). For example, magnetic nanoparticles (composed of a core of iron oxide and a polymeric shell) represent prime candidates for the administration of therapeutic agents in brain tumors (McBain et al., 2008). While the core of iron oxide is responsible for the magnetic retention, the properties of the nanoparticle's surface would determine the interactions with the BBB and the surrounding environment. In particular, the positive surface charges would increase nanoparticles' endocytosis but also increase the excretion velocity of the particle (Chertok et al., 2010). Finally, the nano-bubbles are, in turn, capable of delivering drugs without affecting the endothelium (Surendiran et al., 2009).

New molecular properties 
Nanotechnology could potentially add new properties to existing molecules, making them more competitive. In the 2000s, nano-shells were developed. These silica nanoparticles with a metal shell are able to release their contents after having been heated (by external stimulation such as by infrared light), making them possible pharmacological carriers that could be activated at the most appropriate time(Surendiran et al., 2009).

Moreover dendrimers would eventually be able to replace viral vectors. These nanoscale structures are repetitively branched molecules, do not induce unwanted immune responses and are incorporated into cells by endocytosis (Surendiran et al., 2009). For example in a given cell type deficient in a certain protein, they could enable the tranfser of a gene encoding for the missing protein.

Finally, nanotechnology could combine several molecules in one envelope, which could increase the synergy and efficiency of drug combination (Surendiran et al., 2009).

Nanotechnologies targeting the CNS have been primarily developed in Alzheimer's disease (Moghimi, 2011),Parkinson disease (Pardridge, 2006) or brain tumors in which the integrity of the $\mathrm{BBB}$ is compromised (eg in gliomas where the permeability of blood vessels is greatly increased) (Chertok et al., 2010).

The analysis of living organisms

In vivo imaging:

Nanoparticles are new paramagnetic contrast agents used in MRI for better targeted detection through ligands associated with nanoparticles of nanocrystalline oxide ion (MIONs) (Pan et al., 2010; Surendiran et al., 2009). Fullerenes for example, balloon structures of hexagons of carbon atoms, in their endohectal form, constitute good candidates for contrast agents or biotracers (Pan et al., 2010; Surendiran et al., 2009). This area of research is currently developed in the field of Alzheimer's disease for early detection of senile plaques in certain brain regions (Roney et al., 2005). One could imagine in time, their use as early biomarkers of psychiatric disease, or as markers of response to psychotropic treatments.

\section{Metabolome analysis}

In search of a finer analysis, one can also examine the metabolome.

Metabolites are molecules of low molecular weight $(<1 \mathrm{kDa})$ that can act as carriers, substrates or products involved in cellular biochemical cascades, reflecting the activity of upstream gene expression (Duarte, 2011). The abundance of metabolites is also modulated by environmental 
and epigenetic factors (Duarte, 2011). The metabolome, defined as the set of metabolites belonging to a given biological system, expresses with accuracy the cellular function and overall physiological status of an organism at any given time. Metabonomics has also been recognized by the Food and Drug Association (FDA)(Food and Drug Administration) as an interesting approach complementary of other "-omics" sciences $^{1}$ that could help the development of such markers in evaluating the severity of a disease or drug toxicity. This holistic approach, which can detect unexpected changes or hidden biological events, was only made possible thanks to the development of sophisticated analytical platforms based on nuclear magnetic resonance (NMR) (Duarte, 2011). NMR spectroscopy is particularly powerful in profiling the metabolome and allows the detection of a wide range of different metabolites in biological complexes (biofluids, tissues, cells ...) with a minimum of preparation before the study. Nanotechnologies provide here technical support in the metabolome's study. Analysis of exosomes, nano-vesicles found in saliva, for example, is impossible without a resolution as small as the nanometer (Palanisamy et al., 2010).

The metabolome has been most extensively explored in the field of cancer, diabetes, cardiovascular and neurological diseases (Duarte, 2011) but could be useful in psychiatry. It could be applied to the study of the effect of diet on brain metabolism, and of invasion of the body by an infectious agent (without requiring knowledge of the virus genome, which is still necessary in transcriptional analyzes).

The analysis of the metabolome could profoundly alter the early stages of drug development, with the acquisition of pharmacokinetic data of preclinical toxicity.

The modeling of the central nervous system

The human cortex has a very complex organization. Many studies attempt to model the system and the contribution of nanotechnology is not negligible.

Inorganic synapses:

At the synaptic level, nanotechnology has recently made a breakthrough. The Masakazu Aono team has indeed managed to model an inorganic synapse made of $\mathrm{Ag} 2 \mathrm{~S}$ nanoparticles (Ohno et al., 2011). This synapse is endowed with a short-term potentiation (STP) taking into

\footnotetext{
${ }^{1}$ The neologism omics informally refers to a field of study in biology ending in -omics, such as genomics, proteomics or metabolomics. The related suffix -ome is used to address the objects of study of such fields, such as the genome, proteome or metabolome respectively.
} 
account the history of previous stimuli that were applied, a long-term metastable potentiation (LTP), and is capable of retention and implementation (she forgets a figure for the benefit of another)(Ohno et al., 2011). The next issue is that of moving towards the more complex model of neural networks.

\section{Neural networks}

The first statistical models in terms of networks were based on functional statistics that were not transferable from one described system to another and were limited to describing local networks in their one-dimensional connectivity. These models also had the disadvantage of containing many approximations. The development of quantum mechanics has made it possible to develop models based on the mean field approach, by observing only one element of a network they are able to extrapolate to define the behavior of all other network elements. These models did not refer to laws of chance and described larger networks, consisting of small interconnected networks. But these models do not take into account the effect of external stimuli to the system described. Since, so-called "disruptive" approaches, have helped to integrate the concept of environment, and measure the impact of a disruption of the modeled system, based on knowledge of the unperturbed system and of the disturbance alone. Thus these approaches now allow to model larger systems, and to approach the complexity of the central nervous system (Fraiman et al., 2009; Sporns et al., 2004).

To date, these modeling studies have shown that cortical neuronal networks tended to organize into networks with invariant scale. This architecture allows segregation and functional integration leading to the rapid genesis and transfer of information (Sporns et al., 2004). These networks are also endowed with a metastable dynamic and possess a high structural stability, a very dense connectivity, but also a vulnerability to damage, mainly in the case of elimination of their very connected hubs(Sporns et al., 2004). These models could enrich the field of research on focal damage of the central nervous system such as strokes or hemorrhagic processes. They have the added advantage of being based on data from studies in functional MRI, keeping as close as possible to reality, and limiting approximations.

Based on this observation that cortical neural networks tend to organize themselves into networks with invariant scale, researchers have developed two-dimensional assemblies of nanodevices (metal oxides and chalcogenides materials) to model «neuromorphic» said networks i.e networks that recreate neural organization. These hybrid assemblies called CrossNets are composed of molecular interlock switches corresponding to synapses, 
nanowires representative of axons and dendrites, and nanocircuits $\mathrm{CMOL}^{2}$ corresponding to neuron cell bodies (Likharev et al., 2003). But these constructions which spontaneously organise themselves into invariant scale networks, are capable of self-stimulation and can change after a certain time for their own account (Likharev et al., 2003). These studies have the ambition to simulate cortical networks of $10^{10}$ neurons and $10^{14}$ synapses on a silicon surface of $10 \mathrm{~cm}^{2}$, capable of empowering themselves after specific training (Likharev et al., 2003). These spectacular advances now enable to consider one day a model of artificial intelligence, but also of CNS pathologies such as mental illness, and to observe the effect of external disturbances, in order to predict the evolution and complications of the disease or iatrogenic effects of our care.

\section{Discussion}

We have presented the main lines of research on nanomedicine in psychiatry, and found that the latter is currently overlooked in this research area. Indeed, this article is to our knowledge one of the first that suggests the study of nanotechnology in the field of mental illness.

We have seen that nanocarriers have innovative properties relevant to the blood-brain barrier, which has been implicated in the genesis of some mental illnesses like schizophrenia (Stolp and Dziegielewska, 2009). In addition, we saw that these nanotechnologies could provide new information in human in vivo imaging data (animal models are particularly difficult to design in psychiatry), new data on metabolism and the effect of drugs (from the early stages of their development), knowledge on potential pathophysiological mechanisms of mental illnesses ... The field of psycho-immunology seems to be a particularly promising area of research, these new techniques potentially allowing to trace the expression of viral particles that could be one of the triggers of psychiatric illnesses. The more complex quantum modeling could also revolutionize our understanding of mental illness.

Unfortunately, Wagner and colleagues in 2006 underlined that European financial investment in this area were well below those of the USA (Wagner et al., 2006).

These nanoparticles still present with major hurdles, starting with the health risks associated to their use. We can ask if nanotechnology allows getting drugs into the brain more efficiently, what is there to prevent those same constructs from leaving the brain with equal efficiency? Indeed some nanoparticles induce immune reactions of pro-inflammatory

\footnotetext{
${ }^{2}$ An hybrid silicon circuit. See http://rsfq1.physics.sunysb.edu/ likharev/nano/SPb.pdf
} 
responses, excessive production of free radicals, or favor certain respiratory or gastrointestinal illnesses (Surendiran et al., 2009). The FDA has also implemented the 'Nanotechnology Task Force', work groups created to study the related risks to nanotechnology (Food and Drug Administration, 08-21-2012). It is therefore essential to be cautious and to teach future physicians about the use and risks of nanotechnology (Pautler and Brenner, 2010).

\section{Conclusion}

If nanotechnologies have been developing for nearly 50 years, it is early days for nanomedicine, and we are still far from seeing a practical application in daily psychiatric practice. It seems important, however, that psychiatrists do not forsake this area of research the promises of which could be decisive in the field of mental illness.

Acknowledgements : no financial disclosure.

\section{REFERENCES}

Chertok, B., David, A.E., Yang, V.C., 2010. Polyethyleneimine-modified iron oxide nanoparticles for brain tumor drug delivery using magnetic targeting and intra-carotid administration. Biomaterials 31, 6317-6324.

Duarte, I.F., 2011. Following dynamic biological processes through NMR-based metabonomics: a new tool in nanomedicine? J Control Release 153, 34-39.

Food and Drug Administration, 08-21-2012. www.fda.org

Fraiman, D., Balenzuela, P., Foss, J., Chialvo, D.R., 2009. Ising-like dynamics in large-scale functional brain networks. Phys Rev E Stat Nonlin Soft Matter Phys 79, 061922.

Likharev, K., Mayr, A., Muckra, I., Turel, O., 2003. CrossNets: high-performance neuromorphic architectures for CMOL circuits. Ann N Y Acad Sci 1006, 146-163.

McBain, S.C., Yiu, H.H., Dobson, J., 2008. Magnetic nanoparticles for gene and drug delivery. Int J Nanomedicine 3, 169-180.

Moghimi, S.M., 2011. Bionanotechnologies for treatment and diagnosis of Alzheimer's disease. Nanomedicine 7, 515-518.

Ohno, T., Hasegawa, T., Tsuruoka, T., Terabe, K., Gimzewski, J.K., Aono, M., 2011. Shortterm plasticity and long-term potentiation mimicked in single inorganic synapses. Nat Mater 10, 591-595.

Palanisamy, V., Sharma, S., Deshpande, A., Zhou, H., Gimzewski, J., Wong, D.T., 2010. Nanostructural and transcriptomic analyses of human saliva derived exosomes. PLoS One 5, e8577.

Pan, D., Carauthers, S.D., Chen, J., Winter, P.M., SenPan, A., Schmieder, A.H., Wickline, S.A., Lanza, G.M., 2010. Nanomedicine strategies for molecular targets with MRI and optical imaging. Future Med Chem 2, 471-490. 
Pardridge, W.M., 2006. Molecular Trojan horses for blood-brain barrier drug delivery. Discov Med 6, 139-143.

Pautler, M., Brenner, S., 2010. Nanomedicine: promises and challenges for the future of public health. Int J Nanomedicine 5, 803-809.

Roney, C., Kulkarni, P., Arora, V., Antich, P., Bonte, F., Wu, A., Mallikarjuana, N.N., Manohar, S., Liang, H.F., Kulkarni, A.R., Sung, H.W., Sairam, M., Aminabhavi, T.M., 2005. Targeted nanoparticles for drug delivery through the blood-brain barrier for Alzheimer's disease. J Control Release 108, 193-214.

Sporns, O., Chialvo, D.R., Kaiser, M., Hilgetag, C.C., 2004. Organization, development and function of complex brain networks. Trends Cogn Sci 8, 418-425.

Stolp, H.B., Dziegielewska, K.M., 2009. Review: Role of developmental inflammation and blood-brain barrier dysfunction in neurodevelopmental and neurodegenerative diseases. Neuropathol Appl Neurobiol 35, 132-146.

Surendiran, A., Sandhiya, S., Pradhan, S.C., Adithan, C., 2009. Novel applications of nanotechnology in medicine. Indian J Med Res 130, 689-701.

Szebeni, J., 2011. [Nanomedicine: application of nanotechnology in medicine. Opportunities in neuropsychiatry]. Neuropsychopharmacol Hung 13, 15-24.

Wagner, V., Dullaart, A., Bock, A.K., Zweck, A., 2006. The emerging nanomedicine landscape. Nat Biotechnol 24, 1211-1217.

Wang, J., Byrne, J.D., Napier, M.E., DeSimone, J.M., 2011. More effective nanomedicines through particle design. Small 7, 1919-1931.

Wang, Z.H., Wang, Z.Y., Sun, C.S., Wang, C.Y., Jiang, T.Y., Wang, S.L., 2010. Trimethylated chitosan-conjugated PLGA nanoparticles for the delivery of drugs to the brain. Biomaterials 31, 908-915. 